\title{
Open Research Awards: a Primer from UKRN
}

Authors (Z-A): Kirsty Merrett, Mitul Mehta, Emily Farran, Robert Darby.

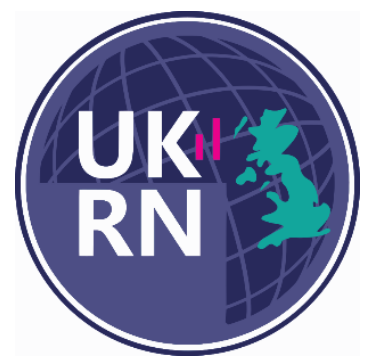

\section{What is an Open Research Award?}

An Open Research Award or Open Research Prize is a competition run by a university to recognise and reward researchers (both staff and students) who have used open practices to make research more accessible, transparent and reproducible. Entry is by means of a case study describing how open practices have been used in a research context or how an activity has developed the environment or infrastructure for Open Research, for example through training activities or the creation of tools. A panel of judges selects finalists, and usually entrants are invited to give presentations at a public event celebrating Open Research. A winner and runners-up are selected with predetermined and transparent marking criteria, with cash prizes given.

Competitions on similar models have been run in the UK by King's College London, the University of Bristol, the University of Reading, and the University of Surrey, and in the Netherlands by the University of Groningen.

\section{Why run an Open Research Award competition?}

The 'publish or perish' research culture puts research integrity in competition with career success. Open Research practices represent a vehicle for change. They support the transparency of research methods, add to the quality and robustness of research outputs, and promote research integrity and research improvement. Institutions can contribute to this muchneeded shift in research culture through the messages that they convey to researchers and the activities that they reward.

An Open Research Award competition sends a public message that the university values, recognises and rewards open and reproducible research behaviours. It incentivises the use of open practices and contributes to a gradual transformation of research culture at an institutional level. It is also an effective means to find exemplars who can be recruited as role models and advocates in a broader programme of action.

\section{How do I run an Open Research Award competition?}

Documentation supporting the competitions run by the University of Bristol, King's College London, the University of Reading, and the University of Surrey is available in the document repository. Contact UKRN to contribute your institution's Open Research Award materials to this repository.

\section{Planning}

A minimum lead time of three months between the launch of the competition and the Award event or announcement is advisable. This allows time for promotion, preparation of entries, shortlisting, and for finalists to prepare presentations in advance of the Award event if one is planned. 
You will need a competition working group. Its composition will depend on the state of Open Research activity within the university, but should have a senior member of staff as lead or sponsor, who can champion the competition and present the business case to the relevant university committee. Professional services staff who support Open Research should be involved in the working group.

The working group lead will need to seek approval and funding to run the competition (unless this has preceded formation of the group). Cost will be incurred for prize payments, running an Award event, web content design, and design and printing of certificates, as relevant.

The competition should be organised in the context of an Open Research communications plan which will include other communications and engagement activities. If there are existing prize competitions for research-related excellence, planners will need to consider whether to integrate with these or run a standalone competition, and ensure communications are coordinated for best effect.

The working group will need to identify the members of the Award Panel as early as possible, and ensure they are available to meet for shortlisting. The Panel Chair at the very least should be able to join the event and may be required to present the Awards.

Establishing the Panel is an opportunity to engage those in senior positions within the institution. A PVC for Research might be asked to chair the Panel and senior staff with research responsibilities invited to join. A Panel of five to seven members might be considered suitable, to allow scope for abstentions if conflicts of interest arise. Its composition should be considered carefully to achieve a balance across disciplines. As Panel members may not necessarily have a shared or developed understanding of Open Research, the working group must ensure they are well-briefed. We suggest a member of professional services staff working in Open Research, such as a librarian or research data manager, sit on the Panel, either as a full member or as an advisor.

\section{The rules}

You will need to establish the rules for the competition. You can refer to the guidelines developed by different universities in the document repository for examples. Some things to consider are:

- Define Open Research carefully. Provide examples of eligible practices in the competition guidelines. The concept of Open Research may not be familiar to all, and in our experience is sometimes confused with impact and public engagement activities. Impact and public engagement may provide evidence of benefits generated by Open Research, but there must be a clear causal relationship between those benefits and the open methods, materials or outputs.

- Define eligibility criteria for the case study as clearly as possible. Does the work have to have been undertaken while at the university, or can a new member of staff submit work undertaken elsewhere? Must the work have an associated openly accessible peer-reviewed research output? What about activities that have developed the environment or infrastructure for Open Research, but which may not have a related publication: for example, contribution to a community metadata standard, development of open research software or hardware, or the creation of teaching and training materials to develop Open Research capabilities?

- Establish who is eligible to enter and to receive prizes. Is the competition open to all staff and students, or only to subsets of these, e.g. early career researchers and research students? Are applications allowed from undergraduates or PGTs, or 
professional services staff (e.g. research software engineers) who have contributed to research projects? Are both individual and team entries allowed? Can teams include external members, and are they eligible to receive prizes?

- Decide what prizes will be given and how they will be awarded. Will the winner of the competition or any sub-category only receive a prize, or will runners-up also receive prizes? What will be the value of prizes and how will they be paid? You may want to split the Award into categories, e.g. one for early career researchers (including research students), and one for established researchers/research groups, or into categories such as Widening Reach and Increasing Quality. Will some or all prizes be awarded solely by the Award Panel, or by other methods, e.g. lottery or audience vote?

- Make judgement criteria for shortlisting and selection of winner(s) clear and transparent. These may be a mix of mandatory and desirable criteria. For example, Open Access publication of associated research outputs (Gold or Green) should be mandatory. Others mandatory criteria might include: any primary data that support the research must be deposited in a data repository (and made openly available unless there is a legitimate reason to restrict access) and referenced by means of a data availability statement including DOI from the related publication; any primary outputs (publications, data, code) must be published under an open licence; the entrant must have an ORCID; team roles must be described using the CRediT Contributor Roles Taxonomy. Assessment criteria should consider: relevance and fit to brief; length; and interest and substance. Criteria and assessment instructions must be documented and provided to the Award Panel for reference when shortlisting and deciding the winner(s).

- Construct the entry form. You will need to think about the maximum length for the case study and the required content. A length in the region of 600-700 words is recommended. Content guidance might set out a list of points to be covered and requirements for references. If the case studies are to be published on the University website, you may need to consult with the website/communications teams at your university regarding length and structure of submissions.

\section{Shortlisting and selection of winner(s)}

Shortlisting and selection of winner(s) in accordance with competition rules can start immediately after the closing date. The working group may undertake an initial longlisting to limit the number of case studies that go forward to the Award Panel for consideration, or the Panel may choose to see all entries and shortlist. Longlisting may eliminate all case studies that fail to meet minimum criteria, e.g. the case study is out of scope, the associated research output is not published Open Access, supporting data/code have not been made available under open licence. You may decide to redact as much personal information about the entrant as you can, to enable blind review. Bear in mind that Panel members are donating their time as judges and the burden should be minimised as far as possible.

Ensure that the Panel has a clear understanding of the judgement criteria and applies them consistently. A point scoring matrix may be employed to apply the criteria fairly. The Panel should be provided with written instructions clearly setting out those criteria and the assessment methods. One member of the Panel should have a high level of experience of Open Research practices, to ensure Open Access, FAIR data and Open Research practices are identified correctly.

The Panel may be asked to independently assess the longlisted entries in advance of the meeting, so that scores and assessment comments can be collated beforehand. It should be possible to achieve early consensus on entries to eliminate and identify at least some which 
can be shortlisted. This will leave a middle ground and the final ranking open for discussion. Where consensus cannot be reached, the final decision may rest with the Chair.

Submissions that do not make the shortlist can still be considered for publication on the university website as case studies, if they are deemed of suitable quality.

In advising entrants of decisions, critical feedback should be offered. This can single out good practice and highlight areas for improvement, and may lead entrants to reflect on their own practice and consider how it might be improved. Entrants can also be encouraged to enter the competition next time round when their project outcomes may have matured.

\section{The Award event}

The Award event should consist of an overview of the criteria for inclusion and the shortlisting process, case study presentations by the finalists, judging if assessed on the day (by the Panel, peer vote, or a combination of the two), the announcement of the winner(s) and presentation of the Award/certificates. The Chair of the Award Panel should present the Award where possible. We recommend shortlisted entries are provided a brief summary of the Panel's assessment to highlight the exemplary elements of each case study. The event might also include one or more keynote speakers to add substance and give the context for the Award. If the event is to be recorded and the recording made publicly available (e.g. on YouTube), be sure to gain consent in advance from presenters and attendees.

\section{Following up}

Communications after the event can include: publishing a write-up of the event; making video recordings of the event/presentations available; publishing and promoting the shortlisted case studies (and possibly some of those on the longlist, if considered of suitable quality).

\section{References}

\section{$\underline{\text { CRediT Contributor Roles Taxonomy }}$}

Open Research Award document repository. If you wish to add your institution's Award materials to the repository, please contact UKRN.

\section{(c) (1) (2)}

This work is licensed under a Creative Commons Attribution-ShareAlike 4.0 International License. 